\title{
ESTADO DEL MIP DE Plutella xylostella (L.) (LEPIDOPTERA: PLUTELLIDAE) EN ARGENTINA
}

\author{
Curis, M. C. ${ }^{1 ;}$ BertolaccinI, I. ${ }^{1}$; LutZ, A. ${ }^{1}$ \& Favaro, J.C. ${ }^{1}$
}

\begin{abstract}
RESUMEN
La polilla de las coles (Plutella xylostella) es una plaga cosmopolita que causa intensos daños a cultivos de Brassicaceas en todo el mundo. Las características de esta especie (ciclo corto, muchas generaciones al año, alta tasa de fecundidad), ayudaron a que haya generado resistencia a muchos insecticidas químicos, tanto puros como en mezclas, incluido biológicos como el Bacillus thuringiensis. A pesar de su importancia y de ser muy estudiada en el exterior, en Argentina se ha realizado poca investigación. En el presente artículo se analizan los avances en los conocimientos de esta plaga en Argentina y se dan pautas que ayuden a su manejo
\end{abstract}

Palabras clave: polilla de las coles, manejo.

\begin{abstract}
MIP status of $P . x y l o s t e l l a($ L.) (Lepidoptera: Plutellidae) in Argentina.

Diamond back moth (Plutella xylostella) is a cosmopolitan pest that causes intense damage to Brassicaceae crops, round the world. Features such as the short cycle, many generations per year, high fertility rate, helped that it has acquired resistance to many chemical insecticides, pure and in mixtures, including a biological like Bacillus thuringiensis. Despite its importance and being very studied abroad, in Argentina few studies have been carried out. The article analyzes the progress of the studies of this pest in Argentina, and gives guidelines that help its management.
\end{abstract}

Key words: diamondback moth, management.

1.- Departamento de Producción Vegetal. Facultad de Ciencias Agrarias, Universidad Nacional del Litoral. Kreder 2805. (3080) Esperanza, provincia de Santa Fe, Argentina. Email: mcuris@fca.unl.edu.ar Manuscrito recibido el 23 de septiembre de 2019 y aceptado para su publicación el 30 de octubre de 2019.

Curis, M. C.; Bertolaccini, I.; Lutz, A.; Favaro, J. C. Estado del MIP de P. xylostella (L.) (Lepidoptera: Plutellidae) en Argentina. FAVE - Ciencias Agrarias 18 (1): 7-18. CC BY-NC-SA 4.0 (c)(1) 


\section{C. Curis et al.}

La "polilla de las coles" Plutella xylostella (L.) (Lepidoptera: Plutellidae) es la plaga insectil que más daño causa en cultivos de crucíferas (Brassicaceae) en el mundo y ocasiona pérdidas económicas significativas al $92 \%$ de los productores con un costo estimado de 168 millones de dólares por año (54). Además de ser una especie migratoria, hábito que, en Argentina y Chile fueron reportados por Talekar \& Shelton (52), es cosmopolita e infecta a 39 especies de crucíferas (54). Esta especialización para la ubicación del hospedador, se basa en la presencia de glucosinolatos, compuestos naturales del metabolismo secundario de las plantas presentes en las crucíferas, que cumplen un rol importante en la interacción planta-insecto, al permitir la ubicación el huésped $(24,50)$, estimular la oviposición y el inicio de la alimentación. Ataca importantes cultivos tanto hortícolas como agrícolas (repollo, coliflor, brócoli, rábano, nabo, coles de Bruselas, col china, mostaza, colza, col, berros, col rizada, colza), aunque también se alimenta de especies silvestres, como Raphistrum rugosum (L.), que puede ser un factor de ingreso de la plaga a los cultivos (41) debido a que ayudan al mantenimiento de las poblaciones cuando no hay cultivos disponibles. Fichetti et al. (16) encontraron a esta plaga desarrollándose sobre garbanzo, logrando completar el ciclo, en localidades donde este cultivo se halló asociado a repollo o brócoli.

Este insecto posee como características biológicas el corto período generacional, alta tasa de fecundidad y resistencia a los insecticidas, factores que permiten el éxito de P. xylostella como organismo plaga.

En Argentina, está presente ocasionando daños severos en los cultivos mencionados, pero a pesar de la importancia se han realizado pocas investigaciones relacionados con la biología y la existencia de enemigos naturales, así como sobre el daño causado, su manejo y control.

\section{Daños}

El daño es causado por la alimentación larval ya que si bien son muy pequeñas, suelen ser numerosas. Su presencia se observa mayoritariamente en las hojas alimentándose del tejido internerval. En ataques a plantas pequeñas prefiere posicionarse en las yemas apicales destruyéndolas, con lo cual puede afectar la formación de la cabeza en el repollo, el brócoli y la coliflor. Los principales estudios han sido realizados en colza y en repollo.

Colza (Brassica napus var oleracea L.)

Algunos trabajos fueron realizados en el cultivo de la colza, por integrantes de la Universidad Nacional de Rosario y el INTA. Montero et al. (37), mencionan que "la polilla de las coles" produce severas defoliaciones desde el estado vegetativo hasta la floración. Además, Lietti et al. (30) desarrollaron protocolos de muestreo secuencial para estimar la densidad poblacional de larvas aplicados en dos etapas fenológicas del cultivo, constituyendo una herramienta indispensable para la toma de decisiones dentro de un programa de MIP para ser utilizada en la toma de decisión del control de la plaga. Folcia y Bado (17), concluyeron que el 80\% del consumo de hojas de este cultivo ocurre en el último estadio larval, en trabajos realizados en condiciones controladas.

En cuanto a los enemigos naturales Montero et al. (36) mencionan que los himenópteros parasitoides Diadegma leontiniae (Cresson), Diadromus collaris (Gravenhorst), Conura sp. y Oomyzus sokolowski (Kurdjumov) logran mortandades superiores al $60 \%$ en algunos casos y mencionan que entre los predadores se observó a Polybia 
scutellaris (White) (Vespidae) alimentándose de larvas sobre las inflorescencias de colza. Entre los predadores potenciales se mencionan a representantes de la familia Carabidae: Trirammatus striatulus (Fabricius), Paranortes cordicollis (Dejean) y Argutoridius bonariensis (Dejean) y arañas de las familias Thomisidae, Lycosidae y Oxiopidae.

Con respecto al uso de insecticidas en colza, en Argentina Montero et al. (37) mencionan que se ha determinado una marcada tolerancia de la plaga a algunos principios activos (piretroides, fosforados y carbamatos), incluso utilizados en mezclas, y han detectado la presencia de larvas 24 horas después de realizadas aplicaciones químicas, alcanzando densidades medias de 4,7 individuos/planta, por lo que recomiendan otras opciones para su control, como el uso de insecticidas biológicos.

Cortés et al. (11) ensayaron diferentes insecticidas puros o en mezclas. Concluyeron que el mejor control se logró con el activo clorantraniliprole mientras que el menor con los reguladores de crecimiento, siendo fácil el control cuando el nivel de ataque es bajo y menos eficiente cuando la plaga ya se instaló en el cultivo.

\section{Repollo (B. oleracea var capitata L.)}

Debido a la presencia de glucosinolatos en los representantes de la familia Brassicaceae, el desarrollo de los estados inmaduros de $P$. xylostella depende, entre otros factores, de las plantas hospederas. Ensayos realizados con larvas en diferentes cultivares de repollo mostraron en el híbrido morado (YR Super Red) genera un efecto de no preferencia (antixenosis) evidenciado por una menor oviposición con respecto a los cultivares de coloración blanca (Gloria) en que la supervivencia de las etapas inmaduras fue menor (antibiosis) (5). En el mismo sentido, Vasicek et al. (56) mostraron que las variedades Corazón de Buey y Cabeza de Hierro fueron las elegidas por los insectos al comienzo y al final del ciclo del repollo, respectivamente. En cuanto al efecto de la alimentación en los parámetros biológicos, Girard et al. (20) no encontraron diferencias en la duración total del ciclo (larva-adulto) el cual fue de 20,72 días para el cultivar blanco Globe Master y de 19,37 días para el tipo morado Ruby Perfection.

En estudios realizados acerca de los enemigos naturales Bertolaccini et al. (4) encontraron variaciones entre diferentes años de muestreos. La mortalidad por hongos entomopatógenos fluctuó entre el $11,1 \%$ al $28,4 \%$, en diferentes años de estudio, mientras que el efecto de los parasitoides varió del 60,3\% al 27,7\%. Los principales parasitoides fueron $O$. sokolowski, C. plutellae y D. leontiniae, y esa variación pude deberse a diferencias en las condiciones climáticas. A similares resultados llegaron Espinoza-Gavilanez \& Grilli (13) en la zona hortícola de la ciudad de Córdoba.

Salto et al. (43) ensayaron diferentes tratamientos con insecticidas y medidas de manejo para el control de la plaga: 1- Bacillus thuringiensis var. Kurstaki (DIPEL $\AA$, 1 l.ha-1), 2- Clorpirifos (LORSBAN ${ }^{\circledR}, 150$ cc/100 l de agua) 3- Siembra de ray-grass (200 kg.ha-1) intercalado entre los surcos y crecimiento natural de vegetación, sin competir con el cultivo y 4- Testigo sin tratar. Los tratamientos químicos resultaron favorables para el control de la plaga.

\section{Estudios de diversidad vegetal}

Fernández et al. (14) compararon la abundancia, riqueza y diversidad de insectos herbívoros y benéficos entre un cultivo extensivo (colza) y uno intensivo (brócoli y 
repollo) con manejo agroecológico, tanto en el cultivo como en los bordes con Brassicaseae silvestres. Estos autores señalaron que la mayor abundancia de herbívoros se debió a $P$. xylostella especialmente en la colza, donde la concentración del recurso alimenticio es mucho mayor. En los cultivos hortícolas se hallaron baja abundancia, riqueza y diversidad tanto de herbívoros como de enemigos naturales, debido a que el manejo agroecológico ocasiona poblaciones estables de insectos benéficos lo que también es causa de la baja abundancia de plagas. Si bien las crucíferas silvestres podrían constituir reservorios de plagas de los cultivos, estarían contribuyendo más a la riqueza y diversidad de insectos benéficos. Este hecho se observó en mayor medida en los bordes de las huertas agroecológicas, ya que constituyen refugios que proveen de polen y néctar, como también de insectos presas en momentos donde el cultivo no está todavía presente, o bien ha sido tratado con insecticidas.

Por otro lado, Pérez (39), usando franjas marginales $B$. rapa (=campestris) L. en el cultivo de repollo, no encontró diferencias en el ataque de la plaga, en la cantidad de los parasitoides ni en los parámetros reproductivos, peso y diámetro de las cabezas del repollo.

\section{Parámetros biológicos}

La duración del ciclo depende de factores bióticos y abióticos, por ejemplo, en larvas criadas sobre colza el ciclo demoró en cumplirse 6,3 días, mientras que en mostacilla ( $R$. rugosum) 3,6 días, con diferencias significativas (41), en cambio la duración del ciclo fue similar cuando se ensayaron diferentes híbridos de repollo con una duración de 20,7 y 19,4 días, para los híbridos Globe Master (de color blanco) y Ruby Perfection (de color morado), respectivamente
(20). Estos mismos autores mencionan que no se hallaron diferencias en la duración total de ciclo cuando se varió la temperatura de 17 y $25^{\circ} \mathrm{C}$ (17,1 y 16,7 días, respectivamente). Para Marchioro \& Foerster (33) la temperatura es fundamental en el ciclo de esta plaga ya que puede afectar el número de generaciones y puede ser responsable de las diferencias en niveles de población registrados entre diferentes regiones.

Son fundamentales los estudios de los factores que afectan la dinámica de la población para el desarrollo de estrategias de manejo para controlar P. xylostella (33). Estos autores, en trabajos posteriores (34) concluyeron que ni la temperatura y ni las precipitaciones registradas durante los experimentos de campo influyeron en la abundancia de la plaga. Sin embargo, para Bhagat et al. (7) la gravedad de la incidencia de la polilla de las coles está muy influenciada por las condiciones climáticas predominantes que varían de región a región. La información básica de las especies de insectos de un agroecosistema y sus densidades de población durante el ciclo del cultivo son indispensables para planificar medidas oportunas para controlar los insectos fitófagos que logren minimizar las pérdidas económicas para el productor.

\section{Niveles de daño económico (NDE)}

Los niveles de daño que se manejan corresponden, frecuentemente, a información extranjera, razón por la cual deben tomarse con precaución debido a que las poblaciones de la plaga y las condiciones ambientales, manejo, etc. suelen tener diferencias entre distintos países.

Para el cultivo de colza Fernández et al. (14) mencionan que los controles químicos se efectúan cuando se alcanzan los siguientes umbrales de tratamiento: 
- Estado de cotiledón a 4 hojas: 25 a 33\% de los cotiledones u hojas verdaderas defoliadas.

- Desde primordio floral a inicio de floración: 100 a 150 larvas/m².

- Desde floración a fructificación: de 200 a 300 larvas $/ \mathrm{m}^{2}$.

En brócoli el NDE es de 2-3 larvas por hoja en 10 plantas, mientras que el umbral económico es de 1-2 larvas cada 10 plantas, que es cuando deben iniciarse las medidas de manejo (10).

Los NDE en repollo son variables en la bibliografía del mundo. Para Shelton et al. (48) este cultivo desde el inicio de formación de cabeza puede tolerar 0,5 larvas/ planta, mientras que para Kirby \& Slosser (28) es de 0,3 larvas/planta. La época de formación de cabeza se puede considerar como el período crítico del daño de P. xylostella en repollo. Durante la época de formación de cabeza el nivel de daño económico, para Rueda Pereyra (42) relacionando la pérdida y las poblaciones de la plaga, resulto ser de 0,83 larvas/planta.

Para brócoli y coliflor Bujanos Muñiz et al. (8) recomiendan los siguientes NDE: 0,5 larvas o más por planta desde el trasplante hasta los primeros 45 días, y 0,2 larvas o más por planta desde los 45 días hasta el último corte. Gashawbeza (19) observó que la etapa previa a la formación de la cabeza es el período crítico, para la infestación, lo que sugiere la necesidad de proteger el cultivo durante esta etapa de crecimiento. Como se observa, el NDE en cultivos hortícolas es mucho más bajo que en la colza, ello está dado porque en este tipo de cultivo, no solo se considera el daño en el área foliar, desde el punto de vista de una baja en la tasa fotosintética, sino también la pérdida en la calidad comercial (49).

Revista FAVE - Ciencias Agrarias 18 (2) 2019

\section{Resistencia}

La notable capacidad genética de este insecto ha permitido convertirse en una plaga líder de insectos resistentes a los insecticidas, y por lo tanto de muy difícil control (44, 47). Ha desarrollado mecanismos para desintoxicar o reducir su sensibilidad (58) a 95 ingredientes activos insecticidas, incluidos los principales grupos y se ha convertido en una de las plagas de insectos más resistentes del mundo (18, 45, 58, 59). Meghana et al. (35) indicó que la tasa de evolución de la resistencia en $P$. xylostella varió según los insecticidas y las ubicaciones geográficas. Esta situación obliga a incrementar el número de aplicaciones, lo cual trae aparejado un incremento de los costos y un alto impacto en la contaminación ambiental.

También se han reportado casos de resistencia al insecticida bacteriano Bacillus thuringiensis (46) y resistencia cruzada a diferentes insecticidas. La aparición de poblaciones de P. xylostella con resistencia a los insecticidas sintéticos y biológicos, ha provocado un aumento considerable en la investigación dirigida al desarrollo de tácticas para el Control Integrado de Plagas basadas en parámetros económicos, sociales y ecológicos (12). En P. xylostella, diferentes recursos genómicos están disponibles para diseñar estrategias de biocontrol (23). Vaschetto \& Beccacece (55) consideran que el ARN no codificante en esta plaga podrían desempeñar un papel importante tanto en el desarrollo como en la resistencia a los insecticidas y describen las tecnologías de ingeniería genética que podrían ser utilizadas para diseñar estrategias eficientes de control. Actualmente en el mundo se están desarrollando líneas transgénicas de repollo $(1,27)$, colza (53), nabo $(29,57)$ con amplia resistencia a los insectos. El 30\% de la superficie sembrada con colza, en el mundo, es transgénica. 


\section{Insecticidas}

Para reducir las pérdidas de rendimiento causadas por esta plaga, muchos productores utilizan principalmente el control químico (52) pero a lo largo de los años, ha demostrado ser ineficaz, porque incluso 15 a 20 aplicaciones de insecticidas durante un ciclo de cultivo no han reducido las pérdidas causadas por P. xylostella (38).

El uso de insecticidas de amplio espectro contribuye a la eliminación de los enemigos naturales. Por otro lado, químicos de mayor selectividad (spinosad, avermectina, neonicotinoides y reguladores del crecimiento) poseen bajo impacto sobre la entomofauna benéfica (15), aunque no están registrados en la mayoría de los casos para su uso en estos cultivos. En Argentina, hay varios insecticidas registrados para el control de P. xylostella: clorantraniliprole $(10 \%)+$ lambdacialotrina (5\%) (colza), lufenurón $(5 \%)+$ profenofos (50\%) (colza), deltametrina $(0,75-20 \%)$ (repollo y coliflor), imidacloprid $(10 \%)+$ betaciflutrina $(1,25 \%)$ (brócoli, repollo y coliflor), flubendiamida (48\%) (brócoli, repollo y coliflor) y metomil (21,6-90\%) (col, coliflor, repollo) (9).

Los insecticidas botánicos no solo son efectivos contra las plagas, sino que son más seguro para los enemigos naturales. Begna \& Damtew (3) ensayaron diversos insecticidas botánicos para el control de $P$. xylostella en repollo, concluyendo que todos los tratamientos redujeron el número de población de larvas y aumentaron el rendimiento comercializable. Entre los productos botánicos, el neem fue el mejor tratamiento ya que dio el mayor rendimiento económico

El desarrollo de nuevos insecticidas naturales y sintéticos y un adecuado patrón de rotación de principios activos contribuyen a la reducción de la presión de selección por las plagas, además de un aumento en la vida útil del insecticida y a retrasar el desarrollo de resistencia.

\section{Enemigos naturales}

Los parasitoides atacan la plaga en diferentes etapas de su desarrollo. Es relevante determinar la asociación entre agentes de control biológico y etapas específicas de las plagas para mejorar las estrategias de control biológico. Más de 90 especies han sido reportadas para el control biológico de P. xylostella (21) y solo en Brasilia (Brasil) se hallaron siete especies diferentes en cultivos de repollo (12). En Argentina se mencionan himenópteros de las familias Ichmeumonidae, Braconidae, Eulophidae y Chalcididae (5), pero no se han identificado otras especies que pueden ser importantes en el control biológico, tales como los parasitoides de huevos.

No existen muchos estudios acerca del efecto de hongos entomopatógenos en el país. Sin embargo Montero et al. (37) mencionan que algunas cepas de la plaga ya mostraron tolerancia a la bacteria $B$. thurigiensis. Pese a ello, sigue siendo una alternativa conveniente, incluso en mezclas con insecticidas que le dan mayor efecto de volteo. También estos autores recomiendan el uso de hongos entomopatógenos: Paecilomyces fumosoroseus (Wize) Brown \& Smith, Metarhizium anisopliae, (Metchnikoff), Beauveria bassiana (Bals.) Vuill. e Hirsutilla sp. Bandy; virus poliédricos, reguladores de síntesis de quitina y cultivos transgénicos, aunque éstos últimos no están regulados para su utilización en nuestro país. Bertolaccini et al. (4) mencionan que en repollo la mortalidad por entomopatógenos varío entre los diferentes años de monitoreo de larvas de 11,1\% en 2006 hasta el 21,9\% de mortalidad larval en la 
campaña 2007, debido posiblemente a diferentes condiciones climáticas en ambos años. Estudios realizados en Brasil demostraron que la efectividad en el control de larvas fue del 70 al 96\% con Paecilomyces tenuipes (Peck) Samson, M. anisopliae y B. bassiana (12).

Manfrino et al. (32) mencionan por primera vez en Argentina a las especies Zoophthora radicans (Brefeld) y Metarhizium rileyi (Farl.) Kepler, S. A. Rehner \& Humber, como patógenos de P. xylostella, en cultivos de coliflor y repollo. Profundizar los estudios sobre los agentes de control biológico que ayuden a reducir la población de esta plaga es importante para la implementación de programas de manejo integrado.

\section{Manejo de la plaga}

En Argentina el manejo de P. xylostella en huertas comerciales se basa principalmente en el uso de insecticidas sintéticos. Sin embargo es necesario encarar otras prácticas, que eviten o retrasen la aparición de resistencia, mantener a los enemigos naturales en el medio y disminuir los residuos de agroquímicos.

Tales tácticas podrían incluir cultivos intercalados con especies de una familia diferente a las crucíferas, basadas en la táctica de confusión que produce en esta plaga la localización de glucosinolatos, lo que altera encontrar la planta hospedera de P. xylostella, dando como resultado una oviposición reducida (25). El repollo intercalado con cebolla y tomate produjo un menor daño en la hoja y la cabeza y un mayor rendimiento que los intercalados con pimienta (2). Según Jankowska (26) esta técnica no solo puede tener impacto en la plaga sino que también en el aumento de los parasitoides y sugiere que los efectos de los cultivos intercalados son mejores a largo plazo.
La época de trasplante es importante debido a las condiciones climáticas que pueden favorecer o no a la plaga. En estudios realizados en repollo Pérez (39) determinó que los trasplantes realizados en primavera tuvieron menor ataque de la plaga, debido a que las condiciones le son menos favorables para su desarrollo.

También como técnica de manejo se puede mencionar el uso de cultivos trampa, usando especies más susceptibles a la plaga que el cultivo principal, para reducir el daño y la cantidad de aplicaciones necesarios para producir cultivos económicos ya que, entre otras cosas, alejan a las plagas del cultivo comercial (22) y disminuye el uso de agroquímicos, debido a que se controla a la plaga solo en la trampa.

Las feromonas sexuales de $P$. xylostella ya se han sintetizado, usándose para determinar las infestaciones y los tratamientos (59). En Brasil se comercializan trampas adhesivas Delta con la feromona sexual artificial (Bioplutella), que permite la captura de machos de la polilla y por lo tanto pueden ser utilizadas para el monitoreo de la plaga (12). En Uruguay se realizaron ensayos con feromonas sexuales para el manejo de $P$. xylostella (51) quienes concluyeron que los resultados fueron promisorios y permite el desarrollo de estrategias de manejo sustentable para racionalizar el uso de pesticidas. Estudios a campo en colza fueron realizados por Fernández et al. (15).

La rotación de cultivos, favorece a un período libre de crucíferas que interrumpe el ciclo de reproducción de la plaga.

Es necesario una aplicación eficaz de insecticidas, logrando una buena cobertura de plaguicidas, debido a que las larvas se asientan en los ápices de crecimiento y en el envés de las hojas. Además las plantas contienen abundantes ceras que cubren las 
hojas y evita que los productos químicos se adhieran convenientemente, por lo que se sugiere la incorporación de adherentes para lograr una mayor persistencia de los productos.

La implementación de técnicas de cultivos limpios puede ser un factor importante en el manejo de P. xylostella. Eliminar los restos de cosecha es una práctica eficiente y fácil que permite cortar el ciclo de la plaga y eliminar los individuos inmaduros que continúan desarrollándose.

Los trabajos realizados sobre esta plaga clave de los cultivos de Brassicaceas son muchos en el exterior. Sin embargo en nuestro país, los estudios de toda índole son escasos, a pesar de la importancia de la plaga. Las diferentes cepas de P. xylostella (40) y de sus agentes de control biológico, presentan particularidades en cuanto a biología, tolerancias a factores climáticos, resistencia a insecticidas, etc., razón por la cual son necesarios trabajos de investigación relacionados al comportamiento, biología, niveles de daño, manejo, etc., con el objetivo de aumentar los rendimientos y disminuir el uso de agroquímicos, además de proporcionar una alternativa eficiente, económica y ecológica para el solución de daños causados por esta plaga y así poder obtener éxito en su manejo. Para Machekano et al. (31) la adopción del MIP está principalmente limitada por la falta de paquetes técnicos desarrollados localmente, la falta de apreciación de este concepto por las partes interesadas, las alternativas limitadas al control químico, la escasez de conocimiento sobre el biocontrol, el desajuste climático entre los sitios de origen y liberación de los agentes de biocontrol y la escasa experiencia en investigación y financiación.

\section{AGRADECIMIENTOS}

El soporte financiero de este trabajo fue provisto por la Universidad Nacional del Litoral (Argentina).

\section{BIBLIOGRAFÍA}

1.-ANDERSON, P.; SHARMA, S.R.; SRESTY, T.V.S.; DEVI, S.; BALA, S.; KUMAR, P.S.; PARDHA SARADHI, P.; FRUTOS, R.; ALTOSAAR, I. y ANANDA KUMAR, P. 2005. Transgenic cabbage (Brassica oleracea var. capitata) resistant to Diamondback moth (Plutella xylostella). Indian Journal of Biotechnology 4: 72-77.

2.-ASARE-BEDIAKO, E.; ADDO-QUAYE, A.A. y MOHAMMED, A. 2010. Control of diamondback moth (Plutella xylostella) on cabbage (Brassica oleracea var capitata) using intercropping with non-host crops. American Journal of Food Technology 5 (4) 269- 274.

3.-BEGNA, F. y DAMTEW, T. 2015. Evaluation of four botanical insecticides against diamondback moth, Plutella xylostella L. (Lepidoptera: Plutellidae) on head cabbage in the central rift valley of Ethiopia. Sky Journal of Agricultural Research 4(5): 97-105

4.-BERTOLACCINI, I.; SÁNCHEZ, D.E.; ARREGUI, M.C.; FAVARO, J.C. y THEILER, N. 2011a. Mortality of Plutella xylostella (Lepidoptera, Plutellidae) by parasitoids in the Province of Santa Fe, Argentina. Revista Brasileira de Entomologia 55(3): 454-456.

\section{5.-BERTOLACCINI, I.; SÁNCHEZ, D.; AR-} REGUI, M.C.; FAVARO, J.C. y THEILER, N. 2011b. Supervivencia de Plutella xylostella L. (Lepidoptera, Plutellidae) en diferentes híbridos de Brassica oleracea L. var. capitata. Revista FAVE - Ciencias Agrarias 10 (1-2). 
6.-BERTOLACCINI, I; SÁNCHEZ，D. y ARREGUI, C. 2010. Incidencia de algunos factores naturales de mortalidad de Plutella xylostella (L.) (Lepidoptera: Plutellidae), en el área centro-este de Santa Fe, Argentina. Horticultura Argentina 29 (68): 20-24.

7.-BHAGAT, P.; YK YADU, Y.K. y SHARMA, G.L. 2018. Seasonal incidence and effect of abiotic factors on population dynamics of diamondback moth (Plutella xylostella L.) on cabbage (Brassica oleracea var. capitata L.) crop. Journal of Entomology and Zoology Studies 6(2): 2001-2003.

8.-BUJANOS MUÑIZ, R.; JARILLO, A.M.; DÍAZ ESPINO, L.F.; GÁMEZ VÁZQUEZ, A.J.; ÁVILA PERCHES, M.A.; HERRERA VEGA, R.; DORANTES GONZÁLEZ, J.R.A. y GÁMEZ VÁZQUEZ, F.P. 2013. Manejo integrado de la palomilla dorso de diamante Plutella xylostella (L.) en la región del bajío, México. Instituto Nacional de Investigaciones Forestales, Agrícolas y Pecuarias. Centro de Investigación Regional Centro Campo Experimental Bajío Celaya, México. Folleto Técnico 27. 44pp.

9.-CASAFE. 2017. Guía de Productos Fitosanitarios para la República Argentina 20172019. $18^{\circ}$ Ed. 1200 p.

10.-CHOI, S.W.; LIM, J.R.; KIM, J.H. y LEE, G.J. 2015. Economic threshold for the diamondback moth (Plutella xylostella L. (Lepidoptera: Plutellidae) on leaf broccoli (Brassica oleracea var. botrytis italiana). AGRIS Csince 54 (1): 19-24.

11.-CORTÉS, E.; ROGGERO, M. y MORERO, M. 2012. Evaluación de distintos insecticidas para el control de Plutella xylostella (L). (polilla de las coles) en el cultivo de colza (Brassica napus L.). Hoja de Información Técnica $\mathrm{N}^{\circ}$ 19. INTA. 5 pp.
12.-DE BORTOLI, S.A.; POLANCZYK, R.A.; VACARI, A.M.; DE BORTOLI, C.P. y DUARTE, R.T. 2013. Plutella xylostella (Linnaeus, 1758) (Lepidoptera: Plutellidae): Tactics for integrated pest management in Brassicaceae. En: Weed and pest control-conventional and new challenges. Soloneski, S. \& M. Larramendy (Eds.). 31-51 pp. Disponible https://www.intechopen.com/books/weedand-pest-control-conventional-and-new-challenges/plutella-xylostella-linnaeus-1758-lepidoptera-plutellidae-tactics-for-integrated-pest-management-in- Acceso: 14-08-2019.

13.-ESPINOZA-GAVILANEZ, R. y GRILLI, M.P. 2019. First record of the spatio-temporal variation of Plutella xylostella (Lepidoptera: Plutellidae) and its parasitoids complex in the horticultural area of Córdoba city in central Argentina. Biological Control 133: 1-8

14.-FERNÁNDEZ, C.; LIETTI, M. y MONTERO, G. 2013. Ensambles de insectos herbívoros y benéficos, en Brassicaceae de cultivos y bordes en agroecosistemas extensivos y huertas agroecológicas. II jornadas técnicas de agricultura urbana, Zavalla. DOI: 10.13140/RG.2.2.19398.78407.

15.-FERNÁNDEZ, C.; VIGNAROLI, L.; GONSEBATT, G.; REYES, V.; LEONCELLI, G.; CÁNEPA, M. E.; PIGOZZI, L.; MONTERO, G. y LIETTI, M. 2015. La "polilla de las coles", Plutella xylostella (L.) (Lepidoptera: Plutellidae) y el cultivo de colza. Agromensajes 41: 47-51.

16.-FICHETTI, P.; AVALOS, S.; MAZZUFERI, V. y CARRERS, J. 2009. Lepidópteros asociados al cultivo del garbanzo (Cicer arietinum L.) en Córdoba, Argentina. Boletín de Sanidad Vegetal 35: 49-58.

17.-FOLCIA, A.M. y BADO. S.G. 1996. Aspectos morfológicos, biológicos e ingesta de Plutella xylostella (L.) (Lepidoptera: Plutellidae) Rev. Facultad de Agronomía 16 (3): 173-178. 
18.-FURLONG, M.J.; WRIGHT, M.J. y DOSDALL, L.M. 2013. Diamondback moth ecology and management: problems, progress, and prospects, Annu. Rev. Entomol. 58: 517-541.

19.-GASHAWBEZA, A. 2006. Comparison of yield loss on cabbage from Diamondback moth, Plutella xylostella L. (Lepidoptera: Plutellidae) using two insecticides. Crop Protection 25(9):915-919. DOI: 10.1016/j. cropro.2005.12.001.

20.-GIRARD, F.P.; BERTOLACCINI, I.; ARREGUI, C.; FAVARO, J.C.; CURIS, M.C. y SÁNCHEZ, D. 2012. Efectos de la temperatura y de la dieta sobre parámetros biológicos de la polilla de las coles (Lepidoptera, Plutellidae). Entomotropica 27 (3): 103-109.

21.-GOODWIN, S. 1979. Changes in the numbers in the parasitoid complex associated with the diamondback moth, Plutella $x y$ lostella (L.) (Lepidoptera) in Victoria. Australian Journal of Zoology 27(6): 981-989.

22.-HASHEELA, E.B.S., NDERITU, J. H. y OLUBAYO, F.M. 2010. Evaluation of border crops against Infestation and damage of cabbage by diamondback moth (Plutella xylostella). Tunisian Journal of Plant Protection 5 (1): 99-106.

23.-HONGBO, L.; XIANG, G.; MUHAMMAD, S. NAEEM; DAN, L.; LING, X.; WENFANG, Z.; GUIXIANG, T. y WEIJUN Z. 2010. Transgenic Brassica napus L. lines carrying a two-gene construct demonstrate enhanced resistance against Plutella xylostella and Sclerotinia sclerotiorum. Plant Cell, Tissue and Organ Culture (PCTOC) 106: 143-151. DOI:10.1007/s11240-010-9902-6.

24.-HOPKINS, R.; VAN DAM, $N$ y VAN LOON, J. 2009. Role of glucosinolates in insect-plant relationships and multitrophic interactions. Annu Rev Entomol 54:57-83.
25.-HUANG, B.; SHI, Z. y HOU, Y. 2014. Host selection behavior and the fecundity of Plutella xylostella (Lepidoptera: Plutellidae) on multiple host plants. J Insect Sci. 14: 251. doi: 10.1093/jisesa/ieu113

26.-JANKOWSKA, B. 2010. Effect of intercropping white cabbage with french marigold (Tagetes patula nana) and pot marigold (Calendula officinalis) on diamondback moth (Plutella xylostella L.) population density and it's parasitoid complex. Vegetable Crops Research Bulletin 73:107-117. doi: 10.2478/ v10032-010-0023- $\mathrm{x}$

27.-JIN, R.G.; LIU, Y-B; TABASHNIK, B.E. y BERTHAKUR, D. 2000. Development of transgenic cabbage (Brassica oleracea var. capitata) for insect resistance by Agrobacterium tumefaciens-mediated transformation In Vitro Cell.Dev.Biol.-Plant 36: 231. https:// doi.org/10.1007/s11627-000-0043-1.

28.-KIRBY, R.D. y SLOSSER, J.E. 1984. Composite economic threshold for three lepidopterous pests of cabbage. Journal of Economic Entomology 77 (3): 725-733.

29.-LI, G.; YUE, L.; LI, F.; ZHANG, H.; ZHANG, H.; QIAN, W.; FANGZ, Z.; WU, J.; WANG, X. ZHANGG, S y SUN, R. 2018. Research progress on Agrobacterium tumefaciens-based transgenic technology in Brassica rapa. Horticultural Plant Journal 4(3): 126-132. https://doi.org/10.1016/j.hpj.2018.03.006.

30.-LIETTI, M.; TRUMPER, E.; FERNÁNDEZ, C.; REYES, V.; LEONCELLI, G. Y VIGNAROLI, L. 2014. Plan de muestreo secuencial para larvas de la polilla de las coles, Plutella xylostella (L.), en colza. Primer Simposio de Canola. Passo Fundo. Brasil. 6pp.

31.-MACHEKANO, H.; MVUMI, B.M. y NYAMUKONDIWA, C. 2017. Diamondback moth, Plutella xylostella (L.) in Southern Africa: Research trends, challenges and insights on sustainable management options. Sustainability. (9): 91. doi: 10.3390/su9020091. 
32.-MANFRINO, R.G.; GUTIERREZ, A.C. y LÓPEZ-LASTRA, C.C. 2018. First reports of Zoophthora radicans (Entomophthoraceae) and Metarhizium rileyi (Clavicipitaceae) as pathogens of Plutella xylostella (Lepidoptera: Plutellidae) in Argentina. Revista Mexicana de Biodiversidad 89: 619-624.

33.-MARCHIORO, C.A. y FOERSTER, L.A. 2011. Development and survival of the diamondback moth, Plutella xylostella (L.) (Lepidoptera: Yponomeutidae) as a function of temperature: effect on the number of generations in tropical and subtropical regions. Neotropical Entomology 40(5): 533-541.

34.-MARCHIORO, C.A. y FOERSTER, L.A. 2016. Biotic factors are more important than abiotic factors in regulating the abundance of Plutella xylostella L., in Southern Brazil. Revista Brasileira de Entomologia 60: 328-333.

35.-MEGHANA, C.; JAYAPPA, J.; ASWATHANARAYANA REDDY, N.; DEVAPPA, V.; SRIDHAR, V. Y KATTEGOUDAR, J. 2018. Assessing susceptibility of diamondback moth, Plutella xylostella (Lepidoptera: Plutellidae) population of different geographic region to selected newer insecticides. Journal of Entomology and Zoology Studies, 6(1): 320-327.

36.-MONTERO, G.; VIGNAROLI, L. y LIETTI, M. 2007b. La "polilla de las coles" principal plaga de la colza en el sur de santa fe. Agromensajes 23: 36-44.

37.-MONTERO, G.; VIGNAROLI, L.; CAVAGLIA, S y LIETTI, M. 2017a. Colza, algo nuevo en la región. Agromensajes 22, 1112.

38.-PÉREZ,C.J; ALVARADO P.; NARVÁEZ, C.; MIRANDA, F.; HERNÁNDEZ, L.; VANEGAS, H.; HRUSKA, A. y SHELTON, A.M. 2000. Assessment of insecticide resistance in five insect pests attacking field and vegetable crops in Nicaragua. J Econ Entomol 93: 1779-1787.
39.-PÉREZ, G. 2013. Efecto de franjas marginales de Brassica campestris L. en repollo sobre Plutella xylostella (Lepidoptera: Plutellidae) y sus enemigos naturales. Tesis para optar por el grado de: Magister Scientiae en Cultivos Intensivos (FCA, UNL). 37 pp.

40.-PERRY, K.D; BAKER, G.J.; POWIS, K.J.; KENT, J.K.; WARD, C.M.; SIMON W. y BAXTER, S.W. 2018. Cryptic Plutella species show deep divergence despite the capacity to hybridize. BMC Evol Biol. 18 (77):1-17. doi:10.1186/s12862-018-1183-4

41.-RODRÍGUEZ, S.M.; TROUCHOT, C. y CARRIZO, P.I. 1997. Observaciones biológicas sobre Plutella xylostella (L.) (Lepidoptera: Iponomeutidae) en canola (Brassica napus oleifera) y en mostacilla (Raphistrum rugosum L.All.). Revista Chilena de Entomología 24: 5-8.

42.-RUEDA PEREYRA, A. 1990. Determinación del período crítico de Plutella $x y$ lostella (L.) en el cultivo de repollo (Brassica oleracea L.) durante la época de apante. Instituto Superior de Ciencias Agropecuarias. Tesis de grado. Managua Nicaragua. 35 pp.

43.-SALTO, C.; TABORDA, R.; SCAGLIA, E. y MORALES, Y. 2007. Diferentes métodos de control de Plutella xylostella en cultivo de repollo (Brassica oleracea var. capitata). XXIX Congreso Argentino de Horticultura. Catamarca. Compendio de trabajos e investigaciones. Pp 47. Disponible: http:// rafaela.inta.gov.ar/info/miscelaneas/111/index.htm (Acceso: 9-9-2019)

44.-SANTOS, V.C.; DE SIQUEIRA, H.A.A.; DA SILVA, J.E. y DE FARÍAS, M.J.D.C. 2011. Insecticide resistance in populations of the diamondback moth, Plutella xylostella (L.) (Lepidoptera: Plutellidae), from the state of Pernambuco, Brazil. Neotrop Entomol 40(2): 264-270. 
45.-SARFRAZ, M. y KEDDIE, B.A. 2005: Conserving the efficacy of insecticides against Plutella xylostella (L.) (Lep., Plutellidae). J. Appl. Entomol., 129:149-157.

46.-SARFRAZ, M. 2004. Interaction between diamondback moth and Bacillus thuringiensis. Outlooks Pest Manag. 15: 167-171.

47.-SARFRAZ, M.; DOSDALL L.M. y KEDDIE, B.A. 2005. Evidence for behavioral resistance by the diamondback moth, Plutella xylostella (L.). JEN 129(6). doi: 10.1111/ j.1439-0418.2005.00969.340-341.

48.-SHELTON, A. M.; ANDALORO, J.T. y BARNARD, J. 1982. Effects of cabbage looper, imported cabbageworm and diamondback moth on fresh market and processing cabbage. J. Econ. Entomol. 75: 742-745.

49.-SRINIVASAN, K. y KRISHNA MOORTHY, P.N. 2008. Indian mustard as a trap crop for management of major lepidopterous pests on cabbage. International Journal of Pest Management 37 (1): 26-32. DOI: 10.1016/j. cropro.2005.12.001

50.-SUN, J.; SONDERBY, I.; HALKIER B.; JANDER G. y DE VOS M. 2009. Non-volatile intact indole glucosinolates are host recognition cues for ovipositing Plutella xylostella. J Chem Ecol 35:1427-1436.

51.-TACAIN, J.; PARPAL, F.; ABBATE, S.; SILVA H.; RIBEIRO, A. y HEGUABURU, V. 2016. Synthesis and field evaluation of the sex pheromone of Plutella xylostella (L.) (Lepidoptera: Plutellidae) in canola (Brassica napus L.). Agrociencia 20 (2):61-67.

52.-TALEKAR, N.S. y SHELTON, A.M. 1993. Biology, ecology, and management of the diamondback moth. Annu Rev Entomol 38: 275301.

53.-THOMZIK, J.E. y HAIN, R. 1990. Transgenic Brassica napus plants obtained by cocultivation of protoplasts with Agrobacterium tumefaciens. Plant Cell Reports 9 (5): 233236. https://doi.org/10.1007/BF00232290.
54.-UTHAMASAMY, S; KANNAN, M.; SENGUTTUVAN, K. Y JAYAPRAKASH, S.A. 2011. Status, damage potential and management of diamondback moth, Plutella xylostella (L.) in Tamil Nadu, India. The $6^{\text {th }}$ International workshop on management of the diamondback moth and other crucifer insect pests. Nakhon Pathom (Thailand). 270-279 pp.

55.-VASCHETTO, L.M. У BECCACECE, H.M. 2019. The emerging importance of noncoding RNAs in the insecticide tolerance, with special emphasis on Plutella xylostella (Lepidoptera: Plutellidae). WIREs RNA. https://doi.org/10.1002/wrna.1539

56.-VASICEK, A.; RICCI, M. y LA ROSA F.R. 1997. Aspectos de preferencia alimentaria de Plutella xylostella L. (Lepidoptera: Yponomeutidae) en algunas crucíferas cultivadas. Facultad de Agronomía 18 (1-2):1-5.

57.-WANG, Y.; ZHANG, Y.; WANG, F.; LIU, C. y LIU, K. 2014. Development of transgenic Brassica napus with an optimized cry1C* gene for resistance to diamondback moth (Plutella xylostella). Can. J. Plant Sci. 94: 1501-1506. doi:10.4141/CJPS-2014-099.

58.-ZHANG, S.; ZHANG, X.; SHEN; J.; DONGYANG, L.; WAN, H.; HONG, Y. y JIANHONG, L. 2017. Cross-resistance and biochemical mechanisms of resistance to indoxacarb in the diamondback moth, Plutella xylostella. Pesticide Biochemistry and Physiology 140: 85-89.

59.-ZONG, G.H.; YAN, S.Q.; LIANG, X.M.; WANG, D.Q. y ZHANG, J.J. 2011. Synthesis of the sex pheromone of Plutella xylostella (L.). Chinese Journal of Organic Chemistry 31(12): 2126-2130. 